\title{
Erratum to: Thyroid Hormone Attenuates Vascular Calcification Induced by Vitamin D3 Plus Nicotine in Rats
}

\author{
Jing Zhang • Jin-Rui Chang • Xiao-Hui Duan • \\ Yan-Rong Yu B Bao-Hong Zhang
}

Published online: 14 March 2015

(c) Springer Science+Business Media New York 2015

\section{Erratum to: Calcif Tissue Int (2015) 96:80-87}

DOI 10.1007/s00223-014-9934-8

In the original publication, the authors missed to mention that Jing Zhang and Jin-Rui Chang contributed equally to this work and should be regarded as co-first authors.

The affiliation of one of the co-authors was incorrect. The correct affiliation is given with this erratum.

The online version of the original article can be found under doi:10. 1007/s00223-014-9934-8.

J. Zhang $(\bowtie)$

School of P.E. and Sports Science, Beijing Normal University,

Beijing 100875, China

e-mail: zhangjing@bnu.edu.cn

J.-R. Chang

Institute of Basic Medicine Science, Xi' an Medical University, Xi' an 710021, China

X.-H. Duan · Y.-R. Yu

Laboratory of Cardiovascular Bioactive Molecules, School of Basic Medical Sciences, Peking University, Beijing 100191,

China

B.-H. Zhang $(\bowtie)$

Hospital of Tsinghua University, Beijing 100084, China

e-mail: hongbaozhang@163.com 